\title{
BMJ Open Assessment of the quantity of microorganisms associated with bronchiectasis in saliva, sputum and nasal lavage after periodontal treatment: a study protocol of a randomised controlled trial
}

To cite: Pinto EH, Longo PL, Camargo CCBde, et al. Assessment of the quantity of microorganisms associated with bronchiectasis in saliva, sputum and nasal lavage after periodontal treatment: a study protocol of a randomised controlled trial. BMJ Open 2016;6:e010564. doi:10.1136/bmjopen-2015010564

- Prepublication history for this paper is available online. To view these files please visit the journal online (http://dx.doi.org/10.1136/ bmjopen-2015-010564).

Received 16 November 2015 Revised 19 March 2016 Accepted 22 March 2016

CrossMark

For numbered affiliations see end of article.

Correspondence to Dr Anna Carolina Ratto Tempestini Horliana, Postgraduate Program in Biophotonics Applied to Health Sciences, Universidade Nove de Julho/UNINOVE R. Vergueiro, 235/249, São Paulo CEP 01504-001, Brazil; annacrth@gmail.com

\section{ABSTRACT}

Introduction: The association between periodontal disease (PD) and chronic obstructive pulmonary disease (COPD) has been widely studied, with aspiration of periodontal pathogens being one of the most accepted causal mechanisms for pulmonary exacerbation. Periodontal treatment (PT) was associated with a decrease in these exacerbations. Bronchiectasis is a pulmonary disease that has many similarities to COPD; however, there are no studies correlating this condition to PD thus far. This study will evaluate if PT reduces proinflammatory cytokines in serum and saliva, as well as halitosis and the amount of microorganisms associated with exacerbation of bronchiectasis in saliva, sputum and nasal lavage 3 months after PT.

Methods and analysis: A total of 182 patients with $\mathrm{PD}$ and bronchiectasis will be randomly allocated to group 1 (positive control; scaling and root planing (SRP)+oral hygiene $(\mathrm{OH})$ ) or group 2 (experimental; $\mathrm{SRP}+$ photodynamic therapy+OH). After 3 months, samples of saliva, nasal lavage and sputum will be collected to determine the level of Pseudomonas aeruginosa, Staphylococcus aureus and

Porphyromonas gingivalis by quantitative PCR. This protocol will determine the efficacy of PT in reducing the most likely niches of bronchiectasis exacerbation by comparing pre- and post-treatment microbiology samples. Furthermore, there will be assessment of oral halitosis and verification of inflammatory cytokines in serum and saliva.

Ethics and dissemination: This protocol has been approved by the Research Ethics Committee of Universidade Nove de Julho. Data will be published in a peer-reviewed journal.

Trial registration number: NCT02514226.

\section{Strengths and limitations of this study}

Although some studies have correlated chronic obstructive pulmonary disease (COPD) with periodontal disease, to date, bronchiectasis has not been studied in the dentistry field.

- Other periodontopathogens, such as Tannerella forsythia and Treponema denticola, as well as other pathogens found in the oral biofilm, such as Haemophilus influenzae and Streptococcus pneumoniae, could be quantified, because they have been identified in tracheal aspirate of patients with COPD. However, since this is the first study, only the most representative microorganisms were chosen. Additional samples of this material will be collected for later analyses of these pathogens.

- Despite the reduction in bacteria in periodontal pockets being followed by their decrease in saliva, it would be interesting to perform microbiological evaluation in this niche, because it could be a potential reservoir for lung bacteria. This material will also be collected for later analyses.

\section{INTRODUCTION}

Periodontal disease (PD) has an infectious origin and affects dental supporting tissue, impacting $19 \%$ of the adult Brazilian population. ${ }^{1}$ It has been associated with various systemic diseases, such as diabetes and cardiovascular disease. ${ }^{2}{ }^{3}$ In recent years, an association has been suggested between PD and pulmonary diseases, such as pneumonia, asthma and chronic obstructive pulmonary 
disease (COPD), although the causal relationship between them is not clear. ${ }^{4}$ The most accepted causal mechanism is aspiration of resident bacteria from the periodontal pocket, which could cause or exacerbate lung infections, ${ }^{5}{ }^{6}$ since there is association between COPD and the plaque index, ${ }^{7}$ and PD and COPD have pathophysiological processes in common: connective tissue destruction and exaggerated inflammatory reaction. ${ }^{8}$ Systemic inflammatory markers are amplified for both, especially tumour necrosis factor (TNF) $\alpha$, interleukin (IL)-6 and IL-8. ${ }^{9} 10111213$ However, the levels of inflammatory markers are not similar among studies ${ }^{5}$ suggesting that more studies are necessary. ${ }^{9}$ Although some studies have correlated COPD and PD, bronchiectasis has not been studied in the dentistry field to date. It is a condition with irreversible and permanent abnormal dilation of the bronchi and bronchioles, high morbidity and decreased quality of life. ${ }^{14}$ Bronchiectasis is often misdiagnosed as asthma or COPD because of the similarities of the clinical manifestations, such as cough and sputum production. ${ }^{15}$ The clinical treatment for this condition is based on control and prevention of recurrent acute exacerbations, which, in turn, reduce public health spending. The aetiology of bronchiectasis can vary widely, including congenital genetic defect and idiopathic causes. ${ }^{16}$ The most common extrinsic factor of bronchiectasis is aspiration of infected mucus, ${ }^{6}$ which could be exacerbated by the presence of PD, but there are no previous studies investigating oral health in these patients. Inflammatory markers, such as IL-1, IL-6, IL-8 and $\mathrm{TNF} \alpha$ are increased in bronchiectasis and in PD. ${ }^{171819}$

The most commonly isolated microorganism in infections is Pseudomonas aeruginosa, whereas Staphylococcus aureus, despite not being as common, is related to more complex infections. ${ }^{16} 202122$ The presence of Ps. aeruginosa is associated with a higher number of hospital admissions. ${ }^{22}$ Periodontal treatment (PT) is not able to eliminate all subgingival pathogens because of inaccessible colonisation sites, and, furthermore, periodontopathogens such as Porphyromonas gingivalis can invade periodontal tissues, ${ }^{23} 24{ }^{25}$ and it has been found in infected lung. ${ }^{26} 27$ Also, Ps. aeruginosa and S. aureus have been found in periodontal pockets of patients with COPD. ${ }^{28}$ Antibiotics are relegated to adjuvants in PT, since the risks outweigh the benefits. ${ }^{49} 30$ On the other hand, they are routinely administered in bronchiectasis, selecting resistant bacterial strains ${ }^{23}$ in the whole body, ${ }^{31}{ }^{32}$ including, possibly, the oral microbiome. Macrolides, fluoroquinolones and azalides are long-term antibiotics used for bronchiectasis, and they are effective against oral bacteria. ${ }^{33}{ }^{34}$ Photodynamic therapy (PDT) is an antimicrobial, atoxic alternative for reducing the quantity of microorganisms in inaccessible sites after $\mathrm{PT}^{35}$ with no side effects and no bacterial resistance reported. ${ }^{36}$ PDT is the association of a photosensitiser with light in the presence of oxygen, ${ }^{29}$ producing reactive oxygen species, such as cytotoxic superoxide and hydroxyl radicals. This reaction generates energy, which is transferred to the bacterial oxygen molecules, leading to cell death. ${ }^{23}$

A common observation in patients with bronchiectasis is the presence of halitosis. ${ }^{37} 38$ It is expected that PT could partly solve this. To date it has not been tested if PT alone can eliminate halitosis in these patients or whether the pulmonary disease induces halitosis by itself.

Although bronchiectasis and COPD share very similar systemic clinical and immunological findings, there are no studies on the periodontal status of patients with bronchiectasis, which leaves a knowledge gap in this area. Therefore, the hypothesis to be tested is whether PT will decrease the number of microorganism species associated with bronchiectasis exacerbation in the saliva environment, sputum and nasal lavage. The primary objective of this study is to assess the decrease in the number of microorganisms associated with bronchiectasis exacerbation, as well as reduction of $P$. gingivalis (currently deemed a cornerstone in PD) in saliva, sputum and nasal lavage before PT and 3 months thereafter. The secondary objectives are to evaluate periodontal clinical parameters, halitosis and proinflammatory cytokines (IL-1, IL-6 and IL8, TNF $\alpha$ ) in serum and saliva before PT and 3 months thereafter with and without PDT.

\section{METHODS}

A randomised, controlled, 3-month, single-centre, parallel-group clinical trial was designed according to the CONSORT Statement and is registered at http://www. clinicaltrial.gov. After verbal and written explanation of the study, patients will sign the informed consent form approved by the Research Ethics Committee (1.057.901) of Nove de Julho University (UNINOVE), and a researcher involved in the study (EHP) will enrol the patients. The study will be performed in accordance with the Declaration of Helsinki (revised in Fortaleza, Brazil, 2013.) A total of 182 patients under medical care at InCor-FMUSP with generalised chronic periodontitis ${ }^{39}$ will receive PT at UNINOVE Dental Clinic, from September 2015 to September 2017. Patients with clinical bronchiectasis will be characterised by chronic sputum production and dilated bronchi confirmed by high-resolution thorax CT. The severity of bronchiectasis will be assessed using the FACED score. ${ }^{40}$

\section{Calculation of sample size}

Sample size calculation was performed for the microbiota ( $P$. gingivalis) as the main outcome. A sample size of 91 participants per group was found to be required to detect a between-group difference of $0.7^{41}$ in the amount of bacteria from baseline to 3-month follow-up ( power $=0.8, \alpha=0.05$ ). This predicted difference equates to an effect size of 0.42 , considering an SD of 1.9 for group 2 (experimental group; PDT) and 1.4 for the root-planing group. ${ }^{41}$ 
We have also calculated the sample size for the secondary outcomes. A sample size of 42 participants per group was found to be required to detect a between-group difference of $0.84^{41}$ in TNF $\alpha$ from baseline to the 3-month follow-up (power $=0.8, \alpha=0.05$ ). This predicted difference equates to an effect size of 0.62 , considering an SD of 1.85 for group 2 and 0.49 for group 1 (positive control group; gold standard). A sample size of 22 participants per group was found to be required to detect a between-group difference of $4.95^{41}$ in IL-1 $\beta$ from baseline to the 3-month follow-up (power $=0.8, \alpha=0.05$ ). This predicted difference equates to an effect size of 0.87 , considering an SD of 5.37 for group 2 and 5.98 for group 1. A sample size of 61 participants per group was found to be required to detect a between-group difference of $2.1^{41}$ in IL-6 from baseline to the 3-month follow-up (power=0.8, $\alpha=0.05$ ). This predicted difference equates to an effect size of 0.51 , considering an SD of 4.62 for group 2 and 3.48 for group 1 . A sample size of 32 participants per group was found to be required to detect a between-group difference of $77.48^{41}$ in IL-8 from baseline to the 3-month follow-up (power $=0.8, \alpha=0.05$ ). This predicted difference equates to an effect size of 0.72 , considering an SD of 94.06 for group 2 and 120.33 for group 1. For halitosis, there is no study comparing scaling and root planing (SRP) + PDT with conventional treatment. However, a previous study comparing baseline with post-non-surgical PT (including oral hygiene $(\mathrm{OH})$ instruction) ${ }^{42}$ demonstrated a high intragroup difference, determining an effect size of 0.77 . Therefore, we believe that the sample size for the mean outcome $(n=91)$ will cover the sample size required to detect intergroup difference for halitosis. The sample size calculation was performed on G*Power software (V.3.1.9). ${ }^{43}$

\section{Calibration of clinical examiner}

One researcher (gold standard) will perform periodontal clinical examinations to achieve maximum reproducibility of the measurements. Ten patients with PD will be evaluated at six sites per tooth (mesiovestibular, vestibular, distobuccal, mesial lingual, lingual and distal-lingual) in the whole mouth, except third molars, for probing depth and clinical attachment loss. Patients who undergo these clinical examinations will not be included in the study groups. The intraclass correlation coefficient (ICC) will be calculated to evaluate intraexaminer concordance, which must be higher than 0.9 . Periodontal probes from University of North Carolina (UNC-15) by Hu-Friedy, Chicago, Illinois, USA will be used. Data will be analysed by ICC test. ${ }^{44}$ The results will be expressed in tables, and compliance with the standard examiner must be higher than 0.8 .

\section{Inclusion and exclusion criteria}

Patients must be $>35$ years of age, of either gender, and have $>10$ teeth with chronic moderate PD-that is, more than $15 \%$ of teeth examined with $\geq 4 \mathrm{~mm}$ probing depth
(PD). Patients with clinical bronchiectasis must be characterised by chronic sputum production and dilated bronchi confirmed by high-resolution thorax CT. All patients should be in a stable condition (no exacerbations requiring antibiotic use in the 3 months before and 3 months after PT). Exclusion criteria include smokers or former smokers who have quit less than 5 years previously, pregnant women, phenytoin or cyclosporin users (because they affect periodontal status), those with decompensated systemic diseases requiring prophylactic antibiotic therapy for PT, those who have used oral antiseptics or anti-inflammatory drugs in the past 3 months, or those who have had PT performed in the past 6 months. Patients with other pulmonary (eg, pulmonary cystic fibrosis, asthma) and nose or throat inflammatory diseases will also be excluded.

\section{Randomisation of patients and allocation concealment}

After SRP, a researcher not involved in the study will divide the patients into two groups by randomised order (Microsoft Excel, 2013 version). Then, opaque envelopes randomly containing information about application of PDT or not will be labelled with sequential numbers. The researcher responsible for PT will open the first envelope and perform the procedure written therein. The internal contents will be revealed only after statistical analysis.

\section{Experimental groups and study design}

Patients will be divided into two groups and they will be subjected to different treatments as follows: group 1, positive control group (gold standard; $n=91 ; O H+S R P$ +simulation of PDT); group 2, experimental group $(\mathrm{n}=91 ; \mathrm{OH}+\mathrm{SRP}+\mathrm{PDT}$ with methylene blue and the red laser diode $(\lambda=660 \mathrm{~nm}))$. All participants will receive PT from an experienced specialist with universal curettes (Hu-Friedy) and ultrasound (Ultra Sound Dabi AtlanteProfi Neo US, Ribeirão Preto, Brazil) in a full-mouth manner (figure 1).

No antibiotics or oral antiseptics will be prescribed. The periodontal supportive therapy will be performed 3 months after the end of PT. ${ }^{45}$ After SRP, a researcher not involved in the study will divide the patients into two groups by randomised order (PDT use or not). In group 1, PT will be performed as described above with Therapy XT (see below) turned off as well. In group 2, the PT will be performed identically to that in group 1 . PDT will be administered in periodontal pockets $>4 \mathrm{~mm}$. Methylene blue (Chimiolux 0.005\%; DMC, São Carlos, São Paulo, Brazil) will be applied with a carpule syringe needle (with stop and no bisel) in the depth of periodontal pockets. After $5 \mathrm{~min}$ of application, the red laser diode $(\lambda=660 \mathrm{~nm})$ will be applied with an output power of $100 \mathrm{~mW}$ (Therapy XT; DMC) and $90 \mathrm{~s}$ of exposure-that is, $9 \mathrm{~J}$ in each point. The laser head will be placed in direct contact with the periodontal pocket. Applications will be held in six sites around the tooth in all teeth. To finalise, there will be 1 min of irradiation in 


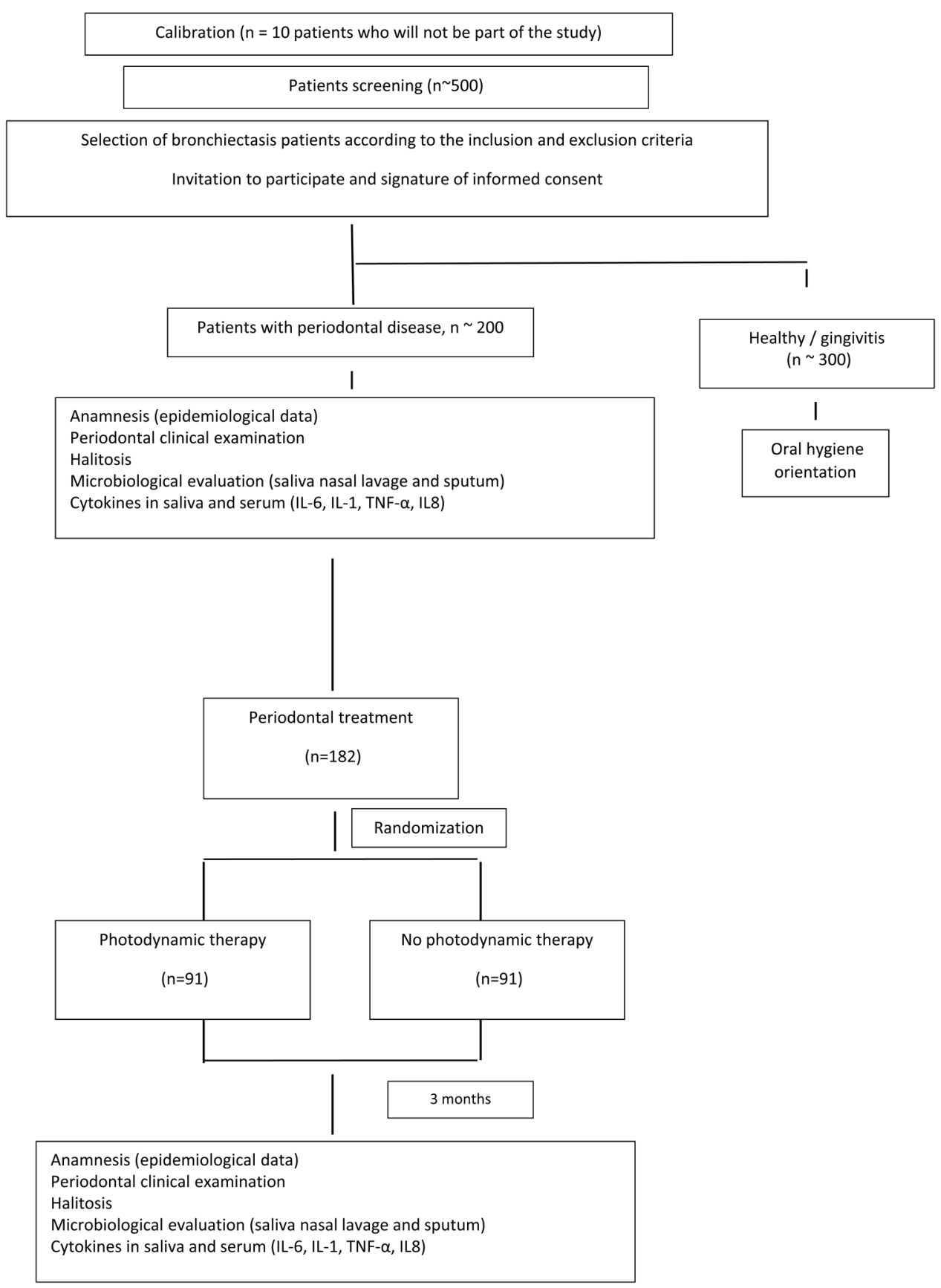

Figure 1 Flow chart summarising the experimental design.

scan around each tooth and rinsing with saline solution to remove the photosensitiser. All the proposed treatments in group $1(\mathrm{OH}+\mathrm{SRP}+$ simulation of $\mathrm{PDT})$ in each patient will be concluded within 1 week. The treatments for group $2(\mathrm{OH}+\mathrm{SRP}+\mathrm{PDT})$ will be also concluded within 1 week. The data analysts who will assess outcome will be blinded during outcome analysis. All possible adverse effects will be noted and qualified during PT and during the maintenance period (3 months) through questionnaires developed for this protocol. The adverse events will be reported in the results section of the manuscript and will be discussed. The Research Ethics Committee of Nove de Julho
University (UNINOVE) will be notified of any protocol alterations. In addition, the records at http://www. clinicaltrial.gov will be notified. Figure 2 summarises the principal points of this protocol.

\section{Sputum, saliva and nasal lavage collection for microbiological evaluation}

Fluid will be kept in the nasal cavities for $10 \mathrm{~s}$ and then washed out by allowing it to flow into a sterile container. The nasal wash will then be transferred to a graduated tube. The nasal lavage will be performed by asking the patient to breathe deeply and hold $10 \mathrm{~mL}$ sterile saline inserted through the nostrils. After collection, RNAlater 
Figure 2 Schematic diagram summarising time points of protocol. IL, interleukin; TNF, tumour necrosis factor.

\begin{tabular}{|c|c|c|c|c|}
\hline & \multicolumn{4}{|c|}{ STUDY PERIOD } \\
\hline & Enrolment & Allocation & Post- allocation & Close-out \\
\hline TIMEPOINT & $-\mathrm{T1}$ & 0 & T1 & $\begin{array}{l}\text { T2 - }(3 \text { months } \\
\text { after periodontal } \\
\text { treatment) }\end{array}$ \\
\hline \multicolumn{5}{|l|}{ ENROLMENT: } \\
\hline Calibration of clinical examiner & $\mathbf{x}$ & & & \\
\hline Informed consent & $\mathrm{x}$ & & & \\
\hline Eligibility screen & $\mathbf{x}$ & & & \\
\hline Allocation & & $\mathbf{x}$ & & \\
\hline \multicolumn{5}{|l|}{ INTERVENTIONS: } \\
\hline G1 - positive control group & & & $\mathbf{x}$ & \\
\hline G2 - experimental group & & & $\mathrm{x}$ & \\
\hline \multicolumn{5}{|l|}{ ASSESMENTS } \\
\hline $\begin{array}{l}\text { Microorganisms' samples in saliva, } \\
\text { sputum and nasal lavage (baseline) }\end{array}$ & $x$ & & & \\
\hline Halitosis (baseline) & $\mathbf{x}$ & & & \\
\hline Cytokines (baseline) & $\mathbf{x}$ & & & \\
\hline $\begin{array}{l}\text { Microorganisms' samples in saliva, } \\
\text { sputum and nasal lavage }\end{array}$ & & & & $x$ \\
\hline Halitosis & & & & $\mathbf{x}$ \\
\hline Cytokines & & & & $\mathrm{x}$ \\
\hline
\end{tabular}

(Qiagen, Valencia, California, USA) will be added to preserve the DNA.

The sputum will be collected in the morning before any food intake. Patients will be asked to breathe deeply several times and then cough deeply; the sample will be collected in a wide-mouth jar. In the laboratory, the sputum specimens will be decontaminated by $\mathrm{N}$ AcetylL-cysteine/ $\mathrm{NaOH}$ centrifuged at $10000 \times g$ for $5 \mathrm{~min}$, and the pellet will be suspended in $50 \mu \mathrm{L}$ buffer solution to extract DNA (1\% Triton X-100, 0.5\% Tween 20, $10 \mathrm{mM}$ Tris/HC1, $\mathrm{pH} 8.0$, and $1 \mathrm{mM}$ EDTA). The material will be sent immediately to the laboratory.

The saliva will be collected and stored exactly as described above. The timeline in figure 3 shows the sequence of procedures and sample collection.

\section{Microbiological evaluation}

Samples will be defrosted and vortexed. DNA extraction will be performed with Master DNA Extraction Kit Pure Kit (Epicentre Technologies, Chicago, Illinois, USA) according to the manufacturer's instructions. Quantitative PCR of the total amount of Ps. aeruginosa, $S$. aureus and $P$. gingivalis will be performed with the SYBR Green System (Applied Biosystems, Foster City, California, USA) in a thermal cycler Step One Plus Real-Time PCR System (Applied Biosystems). This quantification will be performed before treatment and 3 months thereafter to verify the effectiveness of PT and to evaluate if it causes reduction of microbial content of buccal, nasal and lung niches. The outcomes of this protocol will determine the efficacy of PT in reducing the total amount of microorganisms in saliva, sputum and nasal lavage. These outcomes will be classified as (i) better, (ii) no change or (iii) worse than baseline. These results will be compared with a possible reduction of systemic and local inflammation and halitosis after 3 months.

\section{Determination of systemic and saliva inflammatory markers}

Sample collections will be performed through venous puncture by a trained technician at baseline and after 3 months. Sample collections will be centrifuged and serum will be stored. Unstimulated saliva samples will be obtained in sterile collecting tubes and will be kept in a container with ice. All samples will be stored in dry tubes at $-80^{\circ} \mathrm{C}$ until use.

Commercial kits (Peprotech, Rocky Hill, New Jersey, USA) for measuring inflammatory markers (TNF $\alpha$ factor, IL-1, IL- 6 and IL-8) by ELISA will be used according to the manufacturer's instructions.

\section{Halitosis measurements}

Air from inside the oral cavity will be collected with a syringe. With one movement it will be injected into an Oral Chroma (Abilit, Japan), a portable device connected to the computer which captures graph peaks of gas concentration, measuring the thresholds of volatile sulfur compounds (0-1000 parts per billion), after $8 \mathrm{~min}$. To avoid bias, the examination will be conducted in the morning, and participants will be instructed just to brush with water during this day and not to eat food with garlic, onion and hot spices, not to consume alcohol and not to use antiseptic for $48 \mathrm{~h}$ before the oral assessment. They must not have eaten $2 \mathrm{~h}$ before

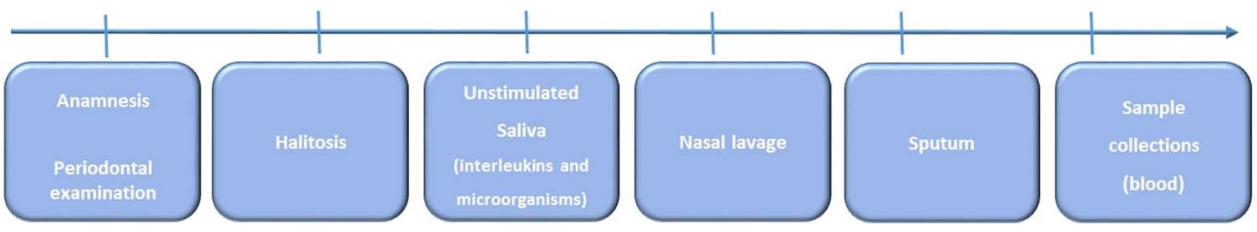

Figure 3 Timeline showing the sequence of procedures and sample collection. 
the test and must not have had coffee, candies or chewing gum or used oral and personal care products with perfume (aftershave, deodorant, perfume and creams).

\section{Data collection and management}

Microbiological collection will always be performed by the same researcher $(\mathrm{CCBdC})$ supervised by a professor (ACRTH), as described under 'Sputum, saliva and nasal lavage collection for microbiological evaluation'. This material will be processed in the Department of Microbiology, Institute of Biomedical Sciences, University of São Paulo, Brazil supervised by two professors (MPAM and PLL). The data will be analysed in an Excel worksheet developed for this purpose.

The collection of saliva to analyse inflammatory markers will be performed by CCBdC supervised by ACRTH, and systemic inflammatory markers will be collected through venous puncture by a single trained technician, as described under 'Determination of systemic and saliva inflammatory markers'. This material will be analysed by the same researcher (CCBdC) in the Laboratory of Biophotonics Applied to Health Sciences, Nove de Julho University, São Paulo, Brazil, supervised by two professors (KPSF and RAMF). The data will be analysed in an Excel worksheet developed for this purpose.

The collection of halitosis will be performed by the same researcher (CCBdC) supervised by SKB. The portable device, Oral Chroma, will be used for halitosis evaluation. Procedures will be performed as previously described. ${ }^{46}$ The air will be injected into the device with a single movement. The Oral Chroma will be connected to a computer (with specific software) allowing the capture of a chart with peaks of gas concentration after 8 min. The data will be analysed in an Excel worksheet developed for this purpose.

In order to improve adherence to the study, we will provide interested patients with information about the study results by mail.

Concerning data management, patients will be identified (ID) after randomisation. Every new datum or piece of material stored (records, documents, samples) will receive the same identifier (eg, ID-1A, related to patient 1 on baseline evaluation; ID-1B, related to patient 1 after 3 months of evaluation).

\section{CT scan}

A CT scan of patients' faces' sinuses for those who have not had a recent one (over 6 months) will be performed. Acquisition of the images will take about 15 min. The CT scan will be used to confirm the diagnosis of the underlying disease (bronchiectasis) and as one of the components for calculating severity criteria (FACED) ${ }^{40}$

\section{Variables of the protocol}

The primary variable will be quantification of $P$ gingivalis, Ps. aeruginosa and $S$. aureus. The amount of microorganisms (measured as log) in saliva, sputum and nasal lavage at baseline will be compared with the amount of the same microorganisms after PT and between groups (group 1 and group 2). These outcomes will be classified as (i) better, (ii) no change or (iii) worse. All variables will be presented as mean \pm SD.

The secondary variables of the study will be quantification of inflammatory cytokines and halitosis. Cytokines will be quantified, $(\mathrm{pg} / \mathrm{mL})$ in saliva and serum. Results will be compared between groups (group 1 and group 2 ) and with baseline. We expect the reduction in local cytokines to be followed by a decrease in systemic ones.

Halitosis will be quantified by measuring the thresholds of volatile sulfur compounds (hydrogen sulfide, methyl mercaptan and dimethyl sulfide). The gas concentrations will be given in standard units of ppd and $\mathrm{ng} / 10 \mathrm{~mL}$. Halitosis will be compared with baseline after PT and between groups (group 1 and group 2). We expect PT to reduce halitosis.

\section{Statistical analysis}

The primary variable will be the quantification of periodontal pathogens and pulmonary microorganisms, and the secondary variables of the study will be quantification of inflammatory cytokines and halitosis. Dental clinical parameters will be assessed at baseline and 3 months after treatment. The Lilliefors test will be used to test the normality of the data. If the data behave normally, the Student t test (Bioestat 5.3, Pará, Brazil) will be used to compare continuous and dependent variables of group 1 and group 2 (quantification of microorganisms, inflammatory cytokines and halitosis). If the data do not behave normally, the Friedman test will be used. $\mathrm{p}<0.05$ will be considered significant. The $\chi^{2}$ test will be used to compare categorical variables between the groups (demographic data) in the comparison between baseline and 3 months. The results will be expressed as mean \pm SD.

\section{Strategies for achieving adequate participant enrolment}

Participants will be recruited in InCor-FMUSP, the hospital where they undergo semi-annual medical care. A researcher (EHP) will perform the periodontal examination in an appropriate room (inside the hospital) and will invite them for PT (after 1 week) in the Dental Clinic of Nove de Julho University, right after periodontal diagnosis. They will be instructed about oral health and be given a set of instructions for performing daily $\mathrm{OH}$.

\section{DISCUSSION}

Bronchiectasis is characterised as a serious lung disease with severe exacerbations, recurrent infections and frequent hospital admissions. ${ }^{14}$ The association between oral biofilm control and severity of lung diseases such as COPD has been demonstrated. ${ }^{7}$ Clinical trials are necessary to confirm whether this premise applies to bronchiectasis. In this study, the conventional PT (gold 
standard) will be compared with treatment with PDT. This alternative treatment was chosen because antibiotics are used more frequently in patients with bronchiectasis than in the general population. ${ }^{47}$ The PDT would provide an additional action, which does not select resistant microorganisms and does not have contraindications. ${ }^{36}$

Several microorganisms, such as T. forsythia, Tr. denticola, P. gingivalis, H. influenza, Ps. aeruginosa, S. aureus and Strep. pneumonia, have been identified in the tracheal aspirate. ${ }^{28}$ Thus, any of them could have been selected as a microorganism indicator of bacterial contamination of saliva, nasal lavage and sputum. We chose to quantify three microorganisms of medical interest. $S$. aureus was chosen because of its high resistance to antimicrobials, ${ }^{48}$ and Ps. aeruginosa was chosen because of its biofilm-forming ability, high resistance to antimicrobials, and high prevalence in bronchiectasis exacerbation. ${ }^{48} 49$ The periodontal pathogen $P$. gingivalis was selected because of its well-established relationship with periodontitis and its association with extra oral infections. ${ }^{24}$ The outcomes of this protocol will determine the efficacy of PT in reducing the total amount of microorganisms in saliva, sputum and nasal lavage. These outcomes will be classified as (i) better, (ii) no change or (iii) worse than baseline. These results will be confronted with a possible reduction in systemic and local inflammation and halitosis after 3 months. Greater improvement in all parameters measured over 3 months is expected for group 1 and group 2. It is expected that group 2 (PDT group) will have better results for periodontal parameters than PT alone after 3 months. ${ }^{50}$

Regarding the other outcomes, such as local and systemic inflammatory cytokines, a slight decrease in local cytokines is expected after PT. Since these patients have a systemic inflammation per se, the reduction in local inflammation is expected to be followed by a slight systemic inflammation. In relation to the halitosis outcome, this is expected to be partly solved after PT, since PD is one of the most probable causes. ${ }^{37} 38$ As mentioned above, to date, it has not been tested if PT alone can eliminate halitosis in these patients or if the pulmonary disease itself induces halitosis.

Maintaining all these outcomes for a 1-2 year follow-up will probably improve lung disease, as demonstrated in patients with COPD. ${ }^{51}$ These patients will be followed (periodontal maintenance) for 2 years and future analyses will be possible.

\footnotetext{
Author affiliations

${ }^{1}$ Biophotonics Applied to Health Sciences, Universidade Nove de Julho, UNINOVE, São Paulo, Brazil

${ }^{2}$ School of Dentistry, Universidade Nove de Julho, UNINOVE, São Paulo, Brazil ${ }^{3}$ Rehabilitation Sciences, Universidade Nove de Julho, UNINOVE, São Paulo, Brazil

${ }^{4}$ Pulmonary Department, Heart Institute (InCor), School of Medicine, University of São Paulo, São Paulo, Brazil

${ }^{5}$ Department of Microbiology, Institute of Biomedical Sciences, University of São Paulo, São Paulo, Brazil
}

Contributors EHP: made substantial contributions to conception and design, acquisition of data, analysis and interpretation of data. PLL: made substantial contributions to conception and design, acquisition of data, analysis and interpretation of data. CCBdC: made substantial contributions to conception and design, acquisition of data, analysis and interpretation of data. SDC: involved in drafting the manuscript and revising it critically for important intellectual content. FDCL: involved in drafting the manuscript and revising it critically for important intellectual content. RS: gave final approval of the version to be published; RA: involved in drafting the manuscript and revising it critically for important intellectual content. KPSF: involved in drafting the manuscript and revising it critically for important intellectual content. MPAM: gave final approval of the version to be published. SKB: involved in drafting the manuscript and revising it critically for important intellectual content. RAMF: involved in drafting the manuscript and revising it critically for important intellectual content. ACRTH: gave final approval of the version to be published.

\section{Competing interests None declared.}

\section{Patient consent Obtained.}

Ethics approval Research Ethics Committee of Universidade Nove de Julho. The results of this study will be presented at international conferences and published in a peer-reviewed journal. Individual data of patients will be protected in order to protect their confidentiality. The identity of the patients will not be disclosed. All data related to the study will be saved in a computer at the Biophotonic Applied to Health Sciences laboratory. A backup will be copied in a cloud locked file with limited access. All laboratory specimens, data collection, reports and forms will be identified by an ID code to maintain confidentiality. All records containing personal identifiers (eg, informed consent forms) will be stored separately from study records and will be identified by code numbers. Only principal investigators (authors of this paper) will be given full access to the cleaned datasets. Project datasets will be saved on the computer of Biophotonics Applied to Health Sciences, Universidade Nove de Julho, UNINOVE, São Paulo, Brazil and all datasets will be protected by password.

Provenance and peer review Not commissioned; externally peer reviewed.

Open Access This is an Open Access article distributed in accordance with the Creative Commons Attribution Non Commercial (CC BY-NC 4.0) license, which permits others to distribute, remix, adapt, build upon this work noncommercially, and license their derivative works on different terms, provided the original work is properly cited and the use is non-commercial. See: http:// creativecommons.org/licenses/by-nc/4.0/

\section{REFERENCES}

1. Brasil. Ministry of Health. Secretariat of Health Care. Secretariat of Health Surveillance. SB Brazil 2010: National Oral Health Survey: main results/Ministry of Health. Secretariat of Health Care. Secretariat of Health Surveillance. Brasília: Ministry of Health, 2012.

2. Preshaw PM, Alba AL, Herrera D, et al. Periodontitis and diabetes: a two-way relationship. Diabetologia 2012;55:21-31.

3. Blaizot A, Vergnes JN, Nuwwareh S, et al. Periodontal diseases and cardiovascular events: meta-analysis of observational studies. Int Dent J 2009;59:197-209.

4. Scannapieco FA, Bush RB, Paju S. Associations between periodontal disease and risk for nosocomial bacterial pneumonia and chronic obstructive pulmonary disease. A systematic review. Ann Periodontol 2003;8:54-69.

5. Azarpazhooh A, Leake JL. Systematic review of the association between respiratory diseases and oral health. $J$ Periodontol 2006;77:1465-82

6. Bansal $\mathrm{M}$, Khatri M, Taneja V. Potential role of periodontal infection in respiratory diseases-a review. J Med Life 2013;6:244-8.

7. Si $\mathrm{Y}$, Fan $\mathrm{H}$, Song $\mathrm{Y}$, et al. Association between periodontitis and chronic obstructive pulmonary disease in a Chinese population. J Periodontol 2012;83:1288-96.

8. Travis J, Pike R, Imamura T, et al. The role of proteolytic enzymes in the development of pulmonary emphysema and periodontal disease. Am J Respir Crit Care Med 1994;150:S143-6.

9. Gan WQ, Man SF, Senthilselvan A, et al. Association between chronic obstructive pulmonary disease and systemic inflammation: a systematic review and a meta-analysis. Thorax 2004;59:574-80. 
10. Finoti LS, Corbi SC, Anovazzi G, et al. Association between IL8 haplotypes and pathogen levels in chronic periodontitis. Eur J Clin Microbiol Infect Dis 2013;32:1333-40.

11. Morelli T, Stella M, Barros SP, et al. Salivary biomarkers in a biofilm overgrowth model. J Periodontol 2014;85:1770-8.

12. Ardila CM, Guzmán IC. Comparison of serum amyloid A protein and C-reactive protein levels as inflammatory markers in periodontitis. J Periodontal Implant Sci 2015;45:14-22.

13. Özer Yücel Ö, Berker E, Mesci L, et al. Analysis of TNF- $\alpha(-308)$ polymorphism and gingival crevicular fluid TNF- $\alpha$ levels in aggressive and chronic periodontitis: A preliminary report. Cytokine 2015;72:173-7.

14. Bustus ME. Bronquiectasia. 10 April 2015. http://www.sbct.org.br/ pdf/livro_virtual/bronquiectasias.pdf

15. Drain M, Elborn JS. Assessment and investigation of adults with bronchiectasis. Assessment and investigation. Eur Respir Mon 2011;52:32-43.

16. Chang $A B$, Bilton D. Exacerbations in cystic fibrosis: 4-Non-cystic fibrosis bronchiectasis. Thorax2008;63:269-76.

17. Tsang KW, Ho PL, Lam WK, et al. Inhaled fluticasone reduces sputum inflammatory indices in severe bronchiectasis. Am J Respir Crit Care Med 1998;158:723-7.

18. Ayhan G, Tas D, Yilmaz I, et al. Relation between inflammatory cytokine levels in serum and bronchoalveolar lavage fluid and gene polymorphism in young adult patients with bronchiectasis. J Thorac Dis 2014:6:684-93.

19. Gale NS, Bolton CE, Duckers JM, et al. Systemic comorbidities in bronchiectasis. Chron Respir Dis 2012;9:231-8.

20. Feldman C. Bronchiectasis: New Approaches to Diagnosis and Management. Clin Chest Med 2011;32:535-46.

21. Aydemir O, Aydemir Y, Ozdemir M. The role of multiplex PCR test in identification of bacterial pathogens in lower respiratory tract infections. J Med Sci 2014;30:1011-16.

22. Zanchet RC, Magalhães AC, Correia AF, et al. A influência de bactérias patogênicas na transportabilidade do escarro e na qualidade de vida de portadores de bronquiectasia. Revista. Brasileira. Fisioterapia 2006;10:457-63.

23. Chawla K, Vishwanath S, Manu MK, et al. Influence of pseudomonas aeruginosa on exacerbation in patients with bronchiectasis. J Glob Infect Dis 2015;7:18-22.

24. Betsy J, Prasanth CS, Baiju KV, et al. Efficacy of antimicrobial photodynamic therapy in the management of chronic periodontitis: a randomized controlled clinical trial. J Clin Periodonto 2014:41:573-81.

25. Mombelli A, Schmid B, Rutar A, et al. Persistence patterns of Porphyromonas gingivalis, Prevotella intermedia/nigrescens, and Actinobacillus actinomycetemcomitans after mechanical therapy of periodontal disease. J Periodontol 2000;71:14-21.

26. Zijlstra EE, Swart GR, Godfroy FJ, et al. Pericarditis, pneumonia and brain abscess due to a combined Actinomyces-actinobacillus actinomycetemcomitans infection. J Infect 1992;25:83-7.

27. Chen AC, Liu CC, Yao WJ, et al. Actinobacillus actinomycetemcomitans pneumonia with chest wall and subphrenic abscess. Scand J Infect Dis 1995;27:289-90.

28. Tan L, Wang H, Li C et.al. 16S rDNA-based metagenomic analysis of dental plaque and lung bacteria in patients with severe acute exacerbations of chronic obstructive pulmonary disease. J Periodontal Res. 2014:49:760-9.

29. Braun A, Dehn C, Krause F, et al. Short-term clinical effects of adjunctive antimicrobial photodynamic therapy in periodontal treatment: a randomized clinical trial. J Clin Periodontol 2008;35:877-84
30. Rams TE, Degener JE, van Winkelhoff AJ. Antibiotic resistance in human chronic periodontitis microbiota. J Periodontol 2014;85:160-9.

31. Conterno L, Fava F, Kieran RV, et al. Obesity and the gut microbiota: does up-regulating colonic fermentation protect against obesity and metabolic disease? Genes Nutr 2011;6: 241-60.

32. Musso G, Gambino R, Cassader M, et al. Obesity, diabetes, and gut microbiota: the hygiene hypothesis expanded? Diabetes Care 2010;33:2277-28

33. ten Hacken $\mathrm{NH}$, Wijkstra PJ, Kerstjens HA. Treatment of bronchiectasis in adults. BMJ 2007;335:1089-93.

34. King PT, Holmes PW. Use of antibiotics in bronchiectasis. Rev Recent Clin Trials 2012;7:24-30.

35. O'Riordan K, Akilov OE, Hasan T. The potential for photodynamic therapy in the treatment of localized infections. Photodiagnosis Photodyn Ther 2005;2:247-62.

36. Konopka K, Goslinski T. Photodynamic therapy in dentistry. J Dent Res 2007;86:694-707.

37. Scully C. Halitosis. BMJ Clin Evid 2014;18:1305.

38. Carmona TI, Posse LJ, Dios PD, et al. Extraoral etiology of halitosis. Med Oral 2001;6:40-7.

39. Armitage GC. Development of a classification system for periodontal diseases and conditions. Ann Periodontol 1999;4:1-6.

40. Martínez-García MÁ, de Gracia J, et al. Multidimensional approach to non-cystic fibrosis bronchiectasis: the FACED score. Eur Respir J 2014:43:1357-67.

41. Kolbe MF, Ribeiro FV, Luchesi VH, et al. Photodynamic therapy during supportive periodontal care: clinical, microbiologic, immunoinflammatory, and patient-centered performance in a split-mouth randomized clinical trial. J Periodontol 2014;85: e277-86.

42. Tsai CC, Chou $\mathrm{HH}, \mathrm{Wu} \mathrm{TL}$, et al. The levels of volatile sulfur compounds in mouth air from patients with chronic periodontitis. J Periodontal Res 2008;43:186-93.

43. Faul F, Erdfelder E, Lang AG, et al. G*Power 3: A flexible statistica power analysis program for the social, behavioral, and biomedical sciences. Behavior Research Methods 2007;39:175-91.

44. Fleiss JL. Design and analysis of clinical experiments. New York: Wiley, 1986.

45. Feres M, Bernal M, Matarazzo F, et al. Subgingival bacterial recolonization after scaling and root planing in smokers with chronic periodontitis. Aust Dent J 2015;60:225-32.

46. Lopes RG, de Godoy CH, Deana AM, et al. Photodynamic therapy as a novel treatment for halitosis in adolescents: study protocol for a randomized controlled trial. Trials 2014;15:443.

47. Welsh EJ, Evans DJ, Fowler SJ, et al. Interventions for bronchiectasis: an overview of Cochrane systematic reviews. Cochrane Database Syst Rev 2015;7:CD010337.

48. King PT, Holdsworth SR, Freezer NJ, et al. Microbiologic follow-up study in adult bronchiectasis. Respir Med. 2007;101:1633-8.

49. Wagner S, Sommer R, Hinsberger S, et al. Novel strategies for the treatment of Pseudomonas aeruginosa infections. J Med Chem 2016. Published Online First: 23 Jan 2016. doi:10.1021/acs. jmedchem.5b01698.

50. Sgolastra F, Petrucci A, Severino M, et al. Adjunctive photodynamic therapy to non-surgical treatment of chronic periodontitis: a systematic review and meta-analysis. J Clin Periodonto 2013;40:514-26.

51. Zhou X, Han J, Liu Z, et al. Effects of periodontal treatment on lung function and exacerbation frequency in patients with chronic obstructive pulmonary disease and chronic periodontitis: a 2-year pilot randomized controlled trial. J Clin Periodontol 2014;41:"564-72. 